\title{
OVERACTIVE BLADDER OR ANXIETY: WHICH CAME FIRST?
}

\author{
Boris Grizelj $^{1}$, Slavko Oreškovićc ${ }^{2,3}$, Mislav Mikuš ${ }^{2,3}$, Vladimir Banovićc ${ }^{2,3}$, \\ Držislav Kalafatić, ${ }^{2,3}$ \& Marina Šprem Goldštajn ${ }^{2,3}$ \\ ${ }^{I}$ Faculty of Medicine, University of Mostar, Mostar, Bosnia and Herzegovina \\ ${ }^{2}$ Department of Obstetrics and Gynecology, University Hospital Center Zagreb, Zagreb, Croatia \\ ${ }^{3}$ School of Medicine, University of Zagreb, Zagreb, Croatia
}

received: 10.5.2021;

revised: 11.7.2021;

accepted: 25.7.2021

\section{SUMMARY}

Objective: Although available diagnostic criteria are intelligible, combination of $O A B$ and anxiety in the same patient presents a perfect example of medical causality dilemma, commonly stated as the question: "which came first: the chicken or the egg?". The aim of this review article is to address available insights in bidirectional association between $O A B$ and anxiety.

Methods: In this review article, we included different types of studies whose results are presented as relative risk (RR) or odds ratio (OR) with a 95\% accuracy. A literature search was conducted with the use of the PubMed and EMBASE electronic databases focusing on identifying articles published in English between 1990 and 2020.

Results: The electronic searches, after duplicate records removal, provided a total of 126 citations. Of these, 107 were excluded after title/abstract screening (not relevant to the review). We examined the full text of 19 publications remaining to summarize possible mechanisms between $O A B$ and anxiety. According to examined literature, our result synthesis provides insight in epidemiology, pathophysiology, diagnostic and therapeutic approach of both conditions.

Conclusion: Temporal relationship between $O A B$ and anxiety is not very well documented because available longitudinal cohort studies are limited. The limitation of the published literature is that most were population-based symptom studies demonstrating high risk of bias. Although data from analysed studies suggest that anxiety and OAB and anxiety might be casually related, studies provided on clinical population are warranted. In addition to the traditional urologic factors, we recommend that psychosocial factors such as anxiety should be assessed routinely in patients with $O A B$.

Key words: overactive bladder - anxiety - pathophysiology - treatment

\section{INTRODUCTION}

According to International Continence Society (ICS), overactive bladder (OAB) presents a condition of "urinary urgency, usually accompanied by frequency and nocturia, with or without urgency urinary incontinence (UUI), in the absence of urinary tract infection (UTI) or other obvious pathology" (Abrams et al. 2003). Based on UUI occurence in overall patients' clinical picture, $\mathrm{OAB}$ can be classified in wet and dry type (Abrams et al. 2003; White and Iglesia 2016). A ratio between wet and $d r y$ type is 1:2, regardless of gender and ethnicity (White and Iglesia 2016). OAB is steadily becoming a significant public-health issue, impacting unfavourably on the quality of life (QoL), performance of daily activities, self-esteem, sleep, sexual function, social life and overall mental health (Bradley et al. 2017; Melotti et al. 2018; Milsom et al. 2014; Tudor et al. 2020; Vrijens et al. 2015). A psychological impact on family members was also present (Bartoli et al. 2010; Kinsey et al. 2016).

The association between affective symptoms and OAB have already been distinguished (Bradley et al. 2017; Kinsey et al. 2016). As claimed by a systematic review of the Dutch authors, $75 \%$ of published studies indicated positive association between anxiety and $\mathrm{OAB}$ (Vrijens et al. 2015). Although clinical presentation of dry $\mathrm{OAB}$ is sometimes unremarkable, anxiety scores were significantly higher in women with confirmed $d r y$ $\mathrm{OAB}$ than in asymptomatic controls (Knight et al. 2012). Estimates of the OAB prevalence in women with diagnosed anxiety vary widely in the published literature, due in part to differing terminologies, diagnostic approaches, and populations studied (Bradley et al. 2017; Ito et al. 2012). Furthermore, the potential for underreporting of $\mathrm{OAB}$ syndrome and anxiety have also impact on establishing exact incidence and prevalence.

On the other hand, with the increasing age of the population worldwide, large socioeconomic impact of OAB is expected (Onukwugha et al. 2009). Considering a cost-utility analysis provided across six European countries, the annual cost-of-illness estimated $€ 7$ billion per year with expected increasing tendency in years to come (Ian Milsom et al. 2014).

Temporal relationship between $\mathrm{OAB}$ and anxiety is not very well documented because available longitudinal cohort studies are limited (Bradley et al. 2017). Although available diagnostic criteria are intelligible, combination of $\mathrm{OAB}$ and anxiety in the same patient presents a perfect example of medical causality dilemma, commonly stated as the question: "which came first: the chicken or the egg?". The aim of this review article is to address available insights in bidirectional association between $\mathrm{OAB}$ and anxiety. 


\section{METHODS}

This review of literature was provided in order to summarize data from relevant articles regarding possible temporal association between $\mathrm{OAB}$ and anxiety.

Considering a nature of this research, an Institutional Review Board permission was not applicable.

\section{Eligibility Criteria}

In this review article, we included different types of studies whose results are presented as relative risk (RR) or odds ratio (OR) with a $95 \%$ accuracy. The results of studies in which the size and characteristics of the observed groups have not been stated were excluded. All included studies were required to describe the number of patients enrolled, type of the study and follow-up period.

The inclusion criteria applied in this review article were the following:

- regarding type of research, we included randomized clinical studies, observational studies, retrospective and prospective studies, cross-sectional and casecontrol studies;

- articles published between 1990-2020;

- in terms of participants, we included women 18-65 years with $\mathrm{OAB}$ diagnosis provided by an urodynamic study in the absence of bacterial infection or obvious pathology;

- anxiety was defined as one of the common anxiety disorders: phobias, followed by social anxiety disorder (SAD), generalized anxiety disorder (GAD) and panic disorder (PD);

- articles written down in English language.

\section{Information Sources and Search Strategies}

A literature search was conducted with the use of the PubMed and EMBASE electronic databases focusing on identifying articles published in English between 1990 and 2020.

Two reviewers (M.Š.G. and M.M.) performed an independent search of sources. The following key words were used: "'overactive bladder", '’anxiety", 'lower urinary tract symptoms", "'panic disorder", ''generalized anxiety disorder", "social anxiety disorder", 'overactive bladder syndrome", "quality of life". The latest date of this search was September $1^{\text {st }}, 2020$.

\section{Study Selection and Data Extraction}

Titles and abstracts were independently screened by two authors (M.Š.G. and M.M.). The study selection performed by two independent reviewers reduced the chance of excluding relevant studies.

The same authors independently assessed studies for inclusion and extracted data about study features, populations selected and outcomes. A manual search of references of included studies was also performed to avoid missing relevant data. Any disagreement or uncertainty were resolved by discussions among the researchers until a consensus was achieved.

We excluded from the analysis case reports/case series and conference abstracts.

The methodological quality of the studies was independently assessed by two investigators using the nine-star Newcastle Ottawa scale (NOS) (Lo et al. 2014). Each study was evaluated based on eight items, categorized into three broad perspectives including selection, comparability, and outcome for cohort studies or exposure for case-control studies. We considered studies with a score of 7 or greater as high quality. Discrepancies were resolved by discussion or through consultation with a third investigator (B.G.).

\section{RESULTS}

The electronic searches, after duplicate records removal, provided a total of 126 citations. Of these, 107 were excluded after title/abstract screening (not relevant to the review). We examined the full text of 19 publications remaining to summarize possible mechanisms between $\mathrm{OAB}$ and anxiety. According to examined literature, our result synthesis provides insight in epidemiology, pathophysiology, diagnostic and therapeutic approach of both conditions. Furthermore, we will address the longitudinal relationship between the $\mathrm{OAB}$ and anxiety.

\section{Epidemiology}

According to a great population-based study conducted in the USA, UK and Sweden, OAB prevalence is ranged between 12 and $22 \%$ and is mainly age-depended (Coyne et al. 2011). The European Prospective Investigation into Cancer and Nutrition (EPIC) study reported the overall prevalence of OAB in Europe and Canada to be $12.8 \%$ among female (Irwin et al. 2006). By contrast, large difference in overall prevalence have been noted in Asia (Lapitan et al. 2001). Such divergence $(53 \%$ vs. $20 \%)$ in reported results between Asia and rest of the world is ensued by using different OAB definition, which was the presence of frequency, urgency, and urge incontinence, either singly or in combination.

According to World Health Organisation (WHO) survey, anxiety remains the most common psychiatric disorder, affecting approximately $20 \%$ of adult population worldwide (Vrijens et al. 2015).

Despite their young age, prevalence in women veterans is slightly above $20 \%$, reflecting relationship between anxiety and $\mathrm{OAB}$ occurrence (Bradley et al. 2017). Furthemore, in published longitudinal study by Bradley and associates, anxiety at the baseline appears to be most predictive of incident OAB, with two-fold greater odds (Bradley et al. 2014). Epidemiological data for Croatian population in terms of OAB prevalence is yet to be determined. 
The importance of affective symptoms in the etiology of OAB have already been emphasized. The upper range of the $\mathrm{OAB}$ prevalence in women with diagnosed anxiety have been demonstrated in a crosssectional study by Melotti and associates; their analysis have shown that $62.4 \%$ of women with $\mathrm{OAB}$ have moderate or severe anxiety (Melotti et al. 2018). OAB and anxiety prevalence are also age-dependent (Kinsey et al. 2016).

\section{Pathophysiology}

According to several proposed hypotheses, OAB presents combination of altered urothelial neurotransmitter release/receptor expression, increase in the amount of pro-inflammatory mediators within the bladder, cross-organ sensitisation and hypothalamic pituitary adrenal (HPA) axis dysregulation (Khasriya et al. 2013; Lai et al. 2016; Merrill et al. 2016).

The importance of periaqueductal gray (PAG) as an integration center for the sensory signals from bladder afferent converge and higher brain centers underlines mutual etiology of OAB and anxiety (Griffiths 2015). Furthermore, patients with $\mathrm{OAB}$ experience significantly higher psychological stress levels comparing to healthy female (Lai et al. 2016). This highlights HPA axis dysfunction as one of the possible pathophysiological patterns in development of both OAB and anxiety (Klausner and Steers 2004). However, expression of symptoms can be determined by genetic and enviromental factors and certain number of clinical trials have described multifactiorial relation between urinary and psychological symptoms while one study suggested bidirectional association between $\mathrm{OAB}$ and anxiety (Bogner et al. 2011). This implicates that social impairment may contribute in promoting $\mathrm{OAB}$ symptoms into more severe form resulting with anxiety occurence (Bradley et al. 2014). Although dry OAB is considered as "symptom-free" form, a study from Knight and associates revealed significantly higher anxiety scores in $\mathrm{OAB} d r y$ study population than in healthy controls (Knight et al. 2012). Their conclusions give us another perspective, representing an underlying response to stress as a mediator for OAB symptoms.

Much better insight in common pathophysiological pathway is provided in recent randomised controlled clinical trials which have demonstrated successful treatment of OAB with antidepressant therapy and vice versa (Kim and Moon 2016; Kinjo et al. 2019; Rogers et al. 2008; Staskin et al. 2007). However, in one study, mirabegron administration provided positive correlation between OAB system score and anxiety, but not depression symptoms (Kinjo et al. 2019). The association between increased urinary frequency and reduction of serotonin levels in the central nervous system have been well documented (Lai et al. 2016). Furthermore, activation of the central serotonergic system with a serotonin uptake inhibitor depresses bladder contractions and increases the micturition threshold volume in animal studies (de Groat 2002).
Up to this point, $\mathrm{OAB}$ and anxiety share similiar biological pathways (serotonin), have shared dysregulation of the HPA axis, increased activity and modulatory levels in anterior cingulate cortex and demonstrate hypoperfusion in prefrontal cortex.

\section{Diagnostic and therapeutic approach}

Although high prevalence rate would suggest the opposite, both $\mathrm{OAB}$ and anxiety are often underreported, underdiagnosed and subsequently undertreated. Patients' reluctance for seeking medical treatment is probably caused by stigmatisation and patients' adoption of $\mathrm{OAB}$ as a part of the normal aging process (Vrijens et al. 2015). Likewise, only $27 \%$ of patients with OAB were receiving treatment in a large population-based study (I. Milsom et al. 2001). Concomitant anxiety in $\mathrm{OAB}$ patients is resulting in even lower medical treatment rate, aggravating overall level of stigma perception (Vrijens et al. 2015). On the other hand, lack of clarity about the definition of both anxiety and OAB may also create difficulties in researching the condition. For example, urgency as essential symptom of OAB, cannot be estimated in objective manner (Kinsey et al. 2016; Wein 2011). Evaluation of patient with both OAB and anxiety is based on an assessment of the degree to which the condition impacts the patients' daily life (White and Iglesia 2016).

Up to this point, there is no conclusive data about urodynamic findings in patients with diagnosed anxiety. Furthermore, it is difficult to determine to what extent anxiety is a contributing factor in urodynamic abnormalities (Sakakibara et al. 2013). Urodynamics in those patients revealed augmented bladder sensitivity, and other variable patterns such as low-compliance detrusor during bladder filling, post-void retention or acontractile detrusor without post-void residual (Sakakibara et al. 2007). Other objective diagnostic approaches in patients with both $\mathrm{OAB}$ and anxiety, such as functional neuroimaging, mostly have experimental character and are not supported in routine clinical surveillance (Grundy et al. 2018). Further studies are warranted in order to clarify inconsistence in the present diagnostic algorithms.

The clear etiology of OAB is not well understood (White and Iglesia 2016). Accordingly, efficacious therapeutic options are limited. The vast majority of available conservative treatment approaches are designed to improve any underlying reversible medical conditions contributing to the syndrome. For instance, control of caffeine and fluid intake, weight loss, surgical treatment in concomitant stress urinary incotinence (SUI), pharmacological treatment of anxiety, optimizing glucose control in diabetes, behavioural interventions and more invasive options are mandatory in OAB therapeutic scheme (Kinjo et al. 2019; Vrijens et al. 2015).

According to published randomized controlled trials, mirabegron, an orally active $\beta_{3}$-adrenoreceptor agonist that causes detrusor relaxation during filling and storage 
phase of micturition is effective in treatment of OAB and anxiety, respectively (Bartoli et al. 2010; Coyne et al. 2011; Kinjo et al. 2019). Even in early phase of mirabegron treatment, loss of anxiety symptoms have been noted (Kinjo et al. 2019).

The effects of antimuscarinics on both urinary and psychological symptoms have been also extensively researched. In their studies, Kim et al. and Staskin et al. demonstrated improvement of the bladder condition and quality of life scores in both gender (Kim and Moon 2016; Staskin et al. 2007). However, a double-blind trial conducted by Rogers et al. have shown positive benefit of anticholinergics compared to placebo on $\mathrm{OAB}$ symptoms, sexual function, and anxiety scores, but not depression scores (Rogers et al. 2008). This results are in line with recently published study on mirabegron effects suggesting that the relief of depression symptoms requires more time than the resolution of $\mathrm{OAB}$ symptoms (Kinjo et al. 2019). Serotonin norepinephrine reuptake inhibitors (SNRIs), although primarily approved for treatment of GAD and depression, are also prescribed for SUI treatment and have also demonstrated benefit on OAB symptoms. Since duloxetine was introduced as an encouraging alternative in the treatment of $\mathrm{OAB}$, beneficial effects on both $\mathrm{OAB}$ and anxiety were reported (Di Rezze et al. 2012; Wang et al. 2015).

Published literature suggests likelihood of successful pharmacological treatment regardless of which primary target is set as a treatment goal - whether it is anxiety or OAB (Kinjo et al. 2019; Vrijens et al. 2015; Wang et al. 2015). In our opinion, and in accordance to published literature, combination of the most tolerable medication and family-based approach incorporated in behavioural training will provide the best treatment outcome.

\section{DISCUSSION}

Although abundant data regarding $\mathrm{OAB}$ and anxiety (including neurobiological mechanisms) argue in favor of a relationship between the two, contemporary studies suggesting that affective factors might also be associated with $\mathrm{OAB}$ are lacking.

In published longitudinal study by Bradley and associates, authors showed that female veterans with anxiety symptoms are more likely to have bothersome urgency incontinence and frequency symptoms (OR 2.7) (Bradley et al. 2014). Their cohort included female veterans who recently returned from deployment to Afghanistan or Iraq and separated from the military. It is important to address that the veteran cohort in mentioned study was mean age of 31 , and there were high rates of other mental health issues $(19 \%$ had post-traumatic stress disorder, $27 \%$ had prior sexual assault) (Bradley et al. 2014).

Another population-based study addressing this issue is the EpiLUTS study, which was an internetbased cross-sectional population survey (Coyne et al.
2011; Sexton et al. 2011). The authors concluded that women who reported urgency and/or urgency incontinence symptoms were more likely to have anxiety than those with minimal symptoms or bother. The results of both presented studies, in accordance of sample characteristics, implicates lack of generalization to the clinical OAB population.

However, the study among the clinical OAB population also demonstrated association between anxiety and OAB - the ORs of having anxiety and moderate/ severe anxiety in $\mathrm{OAB}$ versus healthy controls were 6.0 and 9.2, respectively (Lai et al. 2016).

Regardless of the studied population, data from analysed studies suggest that anxiety and OAB/incontinence might be casually related. This statement have been met on several analyzed levels. According to our analysis, the strength of association between $\mathrm{OAB}$ and anxiety is significant (OR as a main criteria) and consistently demonstrated (Bradley et al. 2014; Felde et al. 2017; Lai et al. 2016; Perry et al. 2006). Furthermore, a strong gradient, dose-response relationship between anxiety and OAB have been observed (Lai et al. 2016). Moreover, a longitudinal, population-based survey studies in the UK and Norway have demonstrated bidirectional temporal relationship (Felde et al. 2017; Perry et al. 2006). In particular, Norwegian 10year longitudinal study have shown that the presence of urgency incontinence at baseline increased the odds of developing anxiety at 10 years, and, conversely, the presence of anxiety at baseline increased the odds of developing urgency incontinence at 10 years (Felde et al. 2017).

Furthermore, there is growing evidence, based primarily on animal studies, that $\mathrm{OAB}$ and anxiety might have shared biological pathways (Lai et al. 2016).

\section{CONCLUSION}

Although $\mathrm{OAB}$ and anxiety are firmly associated, data about whether anxiety improves as a result of treating $\mathrm{OAB}$ and vice versa is inconclusive. The limitation of the published literature is that most were population-based symptom studies demonstrating high risk of bias. Although data from analysed studies suggest that anxiety and $\mathrm{OAB}$ and anxiety might be casually related, studies provided on clinical population are warranted. In light of these data, we recommend that psychosocial factors such as anxiety should be assessed routinely in patients with $\mathrm{OAB}$, in addition to the traditional urologic factors.

Regarding our medical causality dilemma, this medical problem offers two possibilities, neither of which is unambiguously acceptable or preferable. Treatment should be tailored accordingly to dominant symptoms. In this narrative review we address importance of multidisciplinary approach in clinical decision-making, which is unfortunately at this moment in Croatia, inadequate. 


\section{Acknowledgements: None.}

\section{Conflict of interest: None to declare.}

\section{Contribution of individual authors:}

All six authors contribute equally according to the criteria for scientific authorship of the International Committee of Medical Journal Editors (ICMJE):

1. Substantial contributions to conception and design, data collection or analysis, and interpretation of data;

2. Writing of the article or critical review of the intellectual content;

3. Final approval of the version to be published; and

4. Agree to be accountable for all aspects of the work in ensuring that questions related to the accuracy or integrity of any part of the work are appropriately investigated and resolved.

\section{References}

1. Abrams, P., Cardozo, L., Fall, M., Griffiths, D., Rosier, P., Ulmsten, U., et al. (2003). The standardisation of terminology in lower urinary tract function: report from the standardisation sub-committee of the International Continence Society. Urology, 61(1), 37-49. https://doi.org/10.1016/S0090-4295(02)02243-4

2. Bartoli, S., Aguzzi, G., \& Tarricone, R. (2010). Impact on Quality of Life of Urinary Incontinence and Overactive Bladder: A Systematic Literature Review. Urology, 75(3), 491-500. https://doi.org/10.1016/j.urology.2009.07.1325

3. Bogner HR, O'Donnell AJ, de Vries HF, Northington GM \& Joo JH: The temporal relationship between anxiety disorders and urinary incontinence among communitydwelling adults. Journal of Anxiety Disorders 2011; 25:203-208. https://doi.org/10.1016/j.janxdis.2010.09.003

4. Bradley, C. S., Nygaard, I. E., Hillis, S. L., Torner, J. C., \& Sadler, A. G. (2017). Longitudinal associations between mental health conditions and overactive bladder in women veterans. American Journal of Obstetrics and Gynecology, 217(4), 430.e1-430.e8.

https://doi.org/10.1016/j.ajog.2017.06.016

5. Bradley, C. S., Nygaard, I. E., Torner, J. C., Hillis, S. L., Johnson, S., \& Sadler, A. G. (2014). Overactive bladder and mental health symptoms in recently deployed female veterans. The Journal of Urology, 191(5), 1327-1332. https://doi.org/10.1016/j.juro.2013.11.100

6. Coyne, K. S., Sexton, C. C., Kopp, Z. S., Ebel-Bitoun, C., Milsom, I., \& Chapple, C. (2011). The impact of overactive bladder on mental health, work productivity and health-related quality of life in the UK and Sweden: results from EpiLUTS. BJU international, 108(9), 14591471. https://doi.org/10.1111/j.1464-410X.2010.10013.x

7. de Groat, W. C. (2002). Influence of central serotonergic mechanisms on lower urinary tract function. Urology, 59(5 Suppl 1), 30-36. https://doi.org/10.1016/s00904295(01)01636-3

8. Di Rezze S, Frasca V, Inghilleri M, Durastanti V, Cortese A, Giacomelli E \& Millefiorini E: Duloxetine for the treatment of overactive bladder syndrome in multiple sclerosis: a pilot study. Clinical Neuropharmacology 2012; 35:231-234.

https://doi.org/10.1097/WNF.0b013e3182613dce
9. Felde $G$, Ebbesen $M H$ \& Hunskaar S: Anxiety and depression associated with urinary incontinence. A 10year follow-up study from the Norwegian HUNT study (EPINCONT): Anxiety and Depression Associated With Urinary Incontinence. Neurourology and Urodynamics 2017; 36:322-328. https://doi.org/10.1002/nau.22921

10. Griffiths, D. (2015). Neural control of micturition in humans: a working model. Nature Reviews. Urology, 12(12), 695-705. https://doi.org/10.1038/nrurol.2015.266

11. Grundy, L., Caldwell, A., \& Brierley, S. M. (2018). Mechanisms Underlying Overactive Bladder and Interstitial Cystitis/Painful Bladder Syndrome. Frontiers in Neuroscience, 12, 931. https://doi.org/10.3389/fnins.2018.00931

12. Irwin DE, Milsom I, Hunskaar S, Reilly K, Kopp Z, Herschorn $S$ et al.: Population-based survey of urinary incontinence, overactive bladder, and other lower urinary tract symptoms in five countries: results of the EPIC study. European Urology 2006; 50:1306-1314; discussion 13141315. https://doi.org/10.1016/j.eururo.2006.09.019

13. Ito, T., Sakakibara, R., Shimizu, E., Kishi, M., Tsuyuzaki, Y., Tateno, F., et al. (2012). Is Major Depression a Risk for Bladder, Bowel, and Sexual Dysfunction?, 9.

14. Khasriya, R., Sathiananthamoorthy, S., Ismail, S., Kelsey, M., Wilson, M., Rohn, J. L., \& Malone-Lee, J. (2013). Spectrum of bacterial colonization associated with urothelial cells from patients with chronic lower urinary tract symptoms. Journal of Clinical Microbiology, 51(7), 2054-2062. https://doi.org/10.1128/JCM.03314-12

15. Kim, K. S., \& Moon, H. S. (2016). Antimuscarinic Agent Treatment Affecting Patient-Reported Outcomes in Overactive Bladder Syndrome With Depressive Symptoms. International Neurourology Journal, 20(4), 349-355. https://doi.org/10.5213/inj.1624678.339

16. Kinjo M, Yamaguchi T, Tambo M, Okegawa T \& Fukuhara H: Effects of Mirabegron on Anxiety and Depression in Female Patients with Overactive Bladder. Urologia Internationalis 2019; 102:331-335. https://doi.org/10.1159/000497282

17. Kinsey D, Pretorius S, Glover L \& Alexander T: The psychological impact of overactive bladder: A systematic review. Journal of Health Psychology 2016; 21:69-81. https://doi.org/10.1177/1359105314522084

18. Klausner AP \& Steers WD: Corticotropin releasing factor: a mediator of emotional influences on bladder function. The Journal of Urology 2004; 172(6 Pt 2):2570-2573. https://doi.org/10.1097/01.ju.0000144142.26242.f3

19. Knight, S., Luft, J., Nakagawa, S., \& Katzman, W. B. (2012). Comparisons of pelvic floor muscle performance, anxiety, quality of life and life stress in women with dry overactive bladder compared with asymptomatic women. BJU international, 109(11), 1685-1689. https://doi.org/10.1111/j.1464-410X.2011.10590.x

20. Lai, H. H., Shen, B., Rawal, A., \& Vetter, J. (2016). The relationship between depression and overactive bladder/ urinary incontinence symptoms in the clinical $O A B$ population. BMC Urology, 16(1), 60. https://doi.org/10.1186/s12894-016-0179-x

21. Lapitan MC, Chye PL \& Asia-Pacific Continence Advisory Board: The epidemiology of overactive bladder among females in Asia: a questionnaire survey. International Urogynecology Journal and Pelvic Floor Dysfunction 2001; 12:226-231. https://doi.org/10.1007/s001920170043 
22. Lo CK-L, Mertz D \& Loeb M: Newcastle-Ottawa Scale: comparing reviewers' to authors' assessments. BMC medical research methodology 2014; 14:45. https://doi.org/10.1186/1471-2288-14-45

23. Melotti, I. G. R., Juliato, C. R. T., Tanaka, M., \& Riccetto, C. L. Z. (2018). Severe depression and anxiety in women with overactive bladder. Neurourology and Urodynamics, 37(1), 223-228. https://doi.org/10.1002/nau.23277

24. Merrill L, Gonzalez EJ, Girard BM \& Vizzard MA: Receptors, channels, and signalling in the urothelial sensory system in the bladder. Nature Reviews. Urology 2016; 13:193-204. https://doi.org/10.1038/nrurol.2016.13

25. Milsom I, Abrams P, Cardozo L, Roberts RG, Thüroff $J$ $\&$ Wein AJ: How widespread are the symptoms of an overactive bladder and how are they managed? A population-based prevalence study. BJU international 2001; 87:760-766. https://doi.org/10.1046/j.1464-410x. 2001.02228.x

26. Milsom, I, Coyne KS, Nicholson S, Kvasz M, Chen C-I \& Wein AJ: Global Prevalence and Economic Burden of Urgency Urinary Incontinence: A Systematic Review. European Urology 2014; 65:79-95. https://doi.org/10.1016/j.eururo.2013.08.031

27. Onukwugha, E., Zuckerman, I. H., McNally, D., Coyne, K. S., Vats, V., \& Mullins, C. D. (2009). The total economic burden of overactive bladder in the United States: a disease-specific approach. The American Journal of Managed Care, 15(4 Suppl), S90-97.

28. Perry, S., McGrother, C. W., Turner, K., \& Leicestershire MRC Incontinence Study Group. (2006). An investigation of the relationship between anxiety and depression and urge incontinence in women: Development of a psychological model. British Journal of Health Psychology, 11(3), 463-482.

https://doi.org/10.1348/135910705X60742

29. Rogers R, Bachmann G, Jumadilova Z, Sun F, Morrow $J D$, Guan $Z$ \& Bavendam T: Efficacy of tolterodine on overactive bladder symptoms and sexual and emotional quality of life in sexually active women. International Urogynecology Journal 2008; 19:1551-1557. https://doi.org/10.1007/s00192-008-0688-6
30. Sakakibara R, Ito T, Yamamoto T, Uchiyama T, Yamanishi T, Kishi M, et al.: Depression, Anxiety and the Bladder, 2013; 12

31. Sakakibara R, Uchiyama T, Awa Y, Liu Z, Yamamoto T, Ito $T$, et al.: Psychogenic urinary dysfunction: a uro-neurological assessment. Neurourology and Urodynamics 2007; 26:518-524. https://doi.org/10.1002/nau.20321

32. Sexton CC, Coyne KS, Thompson C, Bavendam T, Chen $C-I$ \& Markland A: Prevalence and effect on healthrelated quality of life of overactive bladder in older americans: results from the epidemiology of lower urinary tract symptoms study. Journal of the American Geriatrics Society 2011; 59:1465-1470. https://doi.org/10.1111/j.1532-5415.2011.03492.x

33. Staskin DR, Rosenberg MT, Dahl NV, Polishuk PV \& Zinner NR: Effects of oxybutynin transdermal system on health-related quality of life and safety in men with overactive bladder and prostate conditions: Transdermal oxybutynin for men with OAB. International Journal of Clinical Practice 2007; 62:27-38. https://doi.org/10.1111/j.1742-1241.2007.01625.x

34. Tudor KI, Paši MB \& Musta F: (n.d.). Lower urinary tract symptoms and depression in patients with multiple sclerosis, 8(1), 9

35. Vrijens, D., Drossaerts, J., van Koeveringe, G., Van Kerrebroeck, P., van Os, J., \& Leue, C. (2015). Affective symptoms and the overactive bladder - A systematic review. Journal of Psychosomatic Research, 78(2), 95108. https://doi.org/10.1016/j.jpsychores.2014.11.019

36. Wang $S-M$, Lee $H-K$, Kweon $Y-S$, Lee $C T$ \& Lee $K-U$ : Overactive Bladder Successfully Treated with Duloxetine in a Female Adolescent. Clinical Psychopharmacology and Neuroscience: The Official Scientific Journal of the Korean College of Neuropsychopharmacology 2015; 13:212-214. https://doi.org/10.9758/cpn.2015.13.2.212

37. Wein A: Symptom-based diagnosis of overactive bladder: an overview. Canadian Urological Association Journal 2011; 5(S5):S135-S136. https://doi.org/10.5489/cuaj.11183

38. White $N$ \& Iglesia CB: Overactive Bladder. Obstetrics and Gynecology Clinics of North America 2016; 43:59-68. https://doi.org/10.1016/j.ogc.2015.10.002

Correspondence:

Mislav Mikuš, MD

Department of Obstetrics and Gynecology, University Hospital Center Zagreb

Petrova 13, 10000 Zagreb; Croatia

E-mail:m.mikus19@gmail.com 